\title{
VERIFICATION OF METHOD FOR CALCULATING PARAMETERS OF ACOUSTIC- MAGNETIC DEVICES INSTALLED IN HEAT SUPPLY SYSTEM OF GREENHOUSE COMPLEX
}

\author{
Stefan Onishchenko, Artem Avdzhiyan, Svetlana Korzhakova, Alexey Korzhakov \\ Adyghe State University, Russia \\ stefan.onishchenko@mail.ru,wfrsnkk@gmail.com,korhakov-av@yandex.ru, \\ korzhako-valex@gmail.com
}

\begin{abstract}
The article presents the verification of the method for calculating acoustic-magnetic devices parameters. Periodic greenhouse complex pipe replacement makes a problem of replacing acoustic-magnetic devices. The scientific problem is the design of acoustic-magnetic devices with processing geothermal water optimal efficiency using pipes of various diameters. To calculate the device parameters using the existing methods based on the similarity theory, experimental studies were conducted with five acoustic-magnetic devices for pipes of various diameters on a special test stand. The seven similarity criteria were established and the regression analysis method was used to create a regression equation for the resulting variable - the efficiency of scale formation after geothermal water treatment with an acoustic-magnetic device. Regression equation analysis allows to determine the three significant criteria for ferrite ring properties and geometric characteristics, the acoustic-magnetic device, magnetic and acoustic components. In order to verify the considered methods of calculating the acoustic-magnetic device parameters, experimental studies were carried out according to the experiment plan on the test stand with the acoustic-magnetic device for a pipe with internal diameter of $12 \mathrm{~mm}$. When experimental data were processed, regression analysis methods were used to obtain a multiple regression equation. According to the research results, it was found that indicators of the experimental and control group devices installed on pipes in the greenhouse complex with various values of the internal diameter differ insignificantly. The device's performance indicators that were found experimentally and calculated from the model, also differ insignificantly. This fact proves the efficiency of the method used for calculating parameters in the acoustic-magnetic device design and, in addition, provides an opportunity to create devices with acceptable resulting indicator value for pipes of the required range.
\end{abstract}

Keywords: acoustic-magnetic device (AMD); methods of experiment planning; multiple regression; regression analysis; theory of similarity.

\section{Introduction}

Effective food provision for the population is an actual problem of mankind. Modern greenhouse farms often face the problem of scale formation (solid deposits) on the internal surfaces of the heat supply system equipment. This problem is especially acute with a high salt concentration in geothermal water that is used in a heating system. Intensive scale formation process leads to the decrease of heat transfer or the heating system failure. This problem is solved by the usage of an acoustic-magnetic device that combines two methods of reagentless water treatment into one composite method, in which the liquid is processed by the combined effect of acoustic and magnetic fields. This method is patented in the Russian Federation [1;2]. However, the installed devices are not intended for usage on pipes of various diameters. To solve the scientific problem, a hypothesis is put forward on the possibility of creating acoustic magnetic devices mounted on pipes of various diameters for optimal efficiency of geothermal water processing. For this, it is necessary to calculate the parameters of the acoustic-magnetic device according to the existing technique based on the theory of similarity and conduct experimental studies using five acoustic-magnetic devices for pipes of various diameters. The device parameter calculation and the device design allow to increase the time between routine work during heating system exploitation, and decrease labor and material costs significantly. In order to solve this problem, it is necessary to use the method for calculating the acoustic-magnetic device parameters based on the theory of similarity [3]. The implementation of this method in the heat supply system of a greenhouse complex is possible in case of its verification that determines the research purpose. Thus, the research object is the method for calculating the acousticmagnetic device parameters; the research subject is verification of the acoustic-magnetic device parameter calculating method. To achieve this goal, it is necessary to solve the following tasks:

- to create the laboratory test stand that allows to maintain the necessary process parameters;

- to conduct a series of experiments with five experimental devices installed on pipes of various diameters; 
- to perform statistical processing of experimental data by making a model of the acousticmagnetic treatment process for the resulting variable (the scale formation indicator), highlight significant criteria, check the model for accuracy and adequacy;

- to conduct additional experimental studies on the test stand with the acoustic-magnetic device for the pipe with a specific internal diameter value to obtain a simplified model, and then compare the calculated theoretical values with the experimental data.

\section{Materials and methods}

The use of various empirical coefficients for calculation and comparison of magnetic and acoustic devices depends on the individual characteristics of the experimental conditions and characteristics of the devices. Therefore, the field of application of these coefficients is limited to a narrow range of instrument designs under certain conditions. Significant advantages over various indicators have similarity criteria. Being dimensionless complexes, they are independent of the dimensions of the compared structures [4]. In works [3;5], based on the second and third similarity theorems, the number and composition of the criteria needed to create new devices from previously developed ones are established. However, this technique needs confirmation. For this purpose, a series of experiments were carried out for various types of devices on the test stand (Fig. 1) designed for this purpose.

Experiments were carried out for each of the five acoustic-magnetic devices installed on pipes of various diameters to determine the anti-scale effect. All similarity coefficients were calculated according to the considered method [3]. The ferrite rings used in these devices have the following dimensions: $64 \times 40 \times 9.7 \mathrm{~mm}$; $75 \times 46 \times 12 \mathrm{~mm} ; 75 \times 40 \times 16.8 \mathrm{~mm} ; 64 \times 40 \times 14 \mathrm{~mm} ; 125 \times 80 \times 12 \mathrm{~mm}$. Each of the manufactured devices was installed first on a polypropylene pipe with an internal diameter of $D_{i n}=26 \mathrm{~mm}$, and then with an internal diameter of $D_{i n}=21.2 \mathrm{~mm}$. The choice of pipe diameters is based on the ferrite ring standard size tables.

Process characteristics are recorded during the experiments: the speed of geothermal water movement inside a pipe $\left(v=1 \mathrm{~m} \cdot \mathrm{s}^{-1}\right)$; the temperature of the treated geothermal water $\left(T_{\text {water }}=70^{\circ} \mathrm{C}\right)$.

Values of similarity coefficients and the resulting variable $Y_{r a}$ are obtained as the results of experiments. Values of similarity coefficients $\pi_{62}, \pi_{63}, \pi_{65}, \pi_{60}, \pi_{54}, \pi_{57}$ calculated according to the verified method [3] (Table1).

Values of similarity coefficients and the resulting variable (anti-scale effect)

Table 1

\begin{tabular}{|c|c|c|c|c|c|c|c|c|c|c|}
\hline Ring size & $\boldsymbol{D}_{\mathbf{3 2 i n}}$ & $\boldsymbol{\pi}_{\mathbf{6 2}}$ & $\boldsymbol{\pi}_{\mathbf{6 3}}$ & $\boldsymbol{\pi}_{\mathbf{6 4}}$ & $\boldsymbol{\pi}_{\mathbf{6 5}}$ & $\boldsymbol{\pi}_{\mathbf{6 0}}$ & $\boldsymbol{\pi}_{\mathbf{5 4}}$ & $\boldsymbol{\pi}_{\mathbf{5 7}}$ & $\boldsymbol{Y}_{\boldsymbol{r a}}$ & $\hat{\boldsymbol{Y}}$ \\
\hline $0.064 \cdot 0.009$ & 26.0 & 3.30 & 0.81 & 0.7 & 1.03 & 1.09 & 0.07 & 1.66 & 0.90 & 0.90 \\
\hline $0.064 \cdot 0.009$ & 21.2 & 3.33 & 0.80 & 0.67 & 1.02 & 1.089 & 0.07 & 1.67 & 0.91 & 0.90 \\
\hline $0.075 \cdot 0.012$ & 26.0 & 3.20 & 0.83 & 0.75 & 0.95 & 1.12 & 0.06 & 1.56 & 0.87 & 0.86 \\
\hline $0.075 \cdot 0.012$ & 21.2 & 3.17 & 0.83 & 0.74 & 0.95 & 1.116 & 0.05 & 1.55 & 0.86 & 0.87 \\
\hline $0.075 \cdot 0.017$ & 26.0 & 2.29 & 0.96 & 0.5 & 0.75 & 1.09 & 0.05 & 1.06 & 0.88 & 0.88 \\
\hline $0.075 \cdot 0.017$ & 21.2 & 2.28 & 0.95 & 0.498 & 0.74 & 1.09 & 0.04 & 1.07 & 0.87 & 0.87 \\
\hline $0.064 \cdot 0.014$ & 26.0 & 3.30 & 1.17 & 0.7 & 0.87 & 1.18 & 0.06 & 1.66 & 0.90 & 0.89 \\
\hline $0.064 \cdot 0.014$ & 21.2 & 3.33 & 1.17 & 0.651 & 0.86 & 1.18 & 0.06 & 1.65 & 0.89 & 0.89 \\
\hline $0.125 \cdot 0.012$ & 26.0 & 3.60 & 0.53 & 0.85 & 1.19 & 1.14 & 0.05 & 1.79 & 0.91 & 0.90 \\
\hline $0.125 \cdot 0.012$ & 21.2 & 3.55 & 0.53 & 0.854 & 1.18 & 1.13 & 0.04 & 1.79 & 0.90 & 0.89 \\
\hline
\end{tabular}

Anti-scale effect is used as the optimization parameter. It directly determines the efficiency of liquid treatment in a heat supply system. Its value is defined as follows:

$$
\theta=\frac{M_{O}-M_{H}}{M_{O}},
$$

where $M_{H}$ - mass of solid deposits on the plate during the experiment (when processing liquid);

$M_{0}$ - mass of solid deposits on the plate during the experiment (without liquid treatment).

A multiple regression equation gives an idea of liquid acoustic-magnetic treatment factor interaction. It is based on the obtained results of the experiments. There are many various 
methods [6-9] for making a multiple regression equation. The matrix method of regression analysis is used in the presented research. The regression equation (2) is:

$$
\hat{Y}_{r a}=0.424+0.358 \pi_{62}+0.879 \pi_{63}+0.1270 \pi_{64}+1.600 \pi_{65}-1.132 \pi_{60}-3.017 \pi_{54}-1.026 \pi_{57} .
$$

The Student's coefficient $T_{\beta i}(3)$ is assumed as the criterion for testing the statistical hypothesis $H_{0}$ (the coefficient $\beta_{j}$ is significant):

$$
T \beta_{i}=\frac{\beta_{i}}{S_{b i}}
$$

where $S_{b_{J}}$ - mean-square deviation of the coefficients.

$T_{\beta i}$ has Student's distribution with $n-m$ degrees of freedom. Therefore, the coefficient $\beta_{j}$ significantly differs from zero at the significance level $\alpha$, if:

$$
\left|T_{\beta_{i}}\right|>t_{\alpha, m},
$$

where $t_{\alpha, m}-$ critical value of Student's distribution.

$t_{\alpha, m}=2.2622$. (5)

Confidence intervals of the regression coefficients are:

$\left|T \beta_{0}\right|=0.9937<2.2622 ;\left|T \beta_{1}\right|=1.7276<2.2622 ;\left|T \beta_{2}\right|=2.7377 \geq 2.2622 ;\left|T \beta_{3}\right|=0.8792<2.2622 ;$

$\left|T \beta_{4}\right|=3.7339 \geq 2.2622 ;\left|T \beta_{5}\right|=1.3903<2.2622 ;\left|T \beta_{6}\right|=1.3172<2.2622 ;\left|T \beta_{7}\right|=2.3995 \geq 2.2622$.

The coefficients of criteria $\pi_{63}, \pi_{65}, \pi_{57}$ are significant. Insignificant coefficients with narrow limits of the factor change in the experiment can have a small effect on the optimization parameter, but the factor is not insignificant, so the obtained data need to be confirmed.

After identifying the relevant criteria for the method of verification, it was decided to conduct a series of experiments using another acoustic magnetic device. This device (with ferrite ring $64 \times 46 \times 12 \mathrm{~mm}$ ) was installed on the pipe with the internal diameter value $D_{i n}=12 \mathrm{~mm}$. Experiments were carried out on the test stand according to the experiment plan [10]. The following significant factors are varied: the voltage supplied to the device, the consumed current, the frequency of voltage in the series of experiments.

The significant similarity criteria were calculated for this device, taking into calculation the values of the variable parameters (Tab 2).

Table 2

Factors and variability intervals of the experiment

\begin{tabular}{|c|c|c|c|c|}
\hline \multirow{2}{*}{ Factor level } & \multirow{2}{*}{ Encoded name } & \multicolumn{3}{|c|}{ Factors and their natural values } \\
\cline { 3 - 5 } & & $\boldsymbol{X}_{1}(\boldsymbol{U}), \mathbf{V}$ & $\boldsymbol{X}_{2}(\boldsymbol{I}), \mathbf{A}$ & $\boldsymbol{X}_{3}(\boldsymbol{v}), \mathbf{k H z}$ \\
\hline Basic level & 0 & 12 & 0.1900 & 16 \\
\hline Variability interval & 1 & 2 & 0.0323 & 2 \\
\hline Top level & +1 & 14 & 0.2223 & 18 \\
\hline Bottom level & -1 & 10 & 0.1577 & 14 \\
\hline
\end{tabular}

The scale formation indicator (1) is used as an optimization parameter. There are several different ways to determine it [11-13]. To find it the following method is used: a series of experiments is performed for metal plates placed in untreated geothermal water. The weight method determines the mass of solid deposits formed on the plate surface during the experiment. High-precision electronic scales are used to measure the mass of solid deposits on the metal plate. Next, a series of experiments is repeated using geothermal water treated with the acoustic-magnetic device. The time of experiments for both cases is fixed and the same. Based on the results of the experiments, the regression equation with significant factors $\pi_{63}, \pi_{65}, \pi_{57}$ is obtained. It is necessary to calculate the following matrices for the following equation. Matrix $X$ of the main characteristics of devices (in the form of similarity criteria) and matrix-column $Y$ with the values of the resulting variable (scale formation indicator) (7): 


$$
X=\left[\begin{array}{lll}
1.830 & 0.519 & 1.450 \\
1.830 & 0.519 & 1.442 \\
1.830 & 0.510 & 1.450 \\
1.830 & 0.510 & 1.442 \\
1.820 & 0.519 & 1.450 \\
1.820 & 0.510 & 1.442 \\
1.820 & 0.510 & 1.450 \\
1.820 & 0.510 & 1.442
\end{array}\right], Y=\left[\begin{array}{l}
0.88 \\
0.89 \\
0.87 \\
0.88 \\
0.88 \\
0.88 \\
0.88 \\
0.87
\end{array}\right] .
$$

To obtain the values of the regression coefficients of the equation, the inverse matrix $\left(X^{T} X\right)^{-1}$ must be multiplied by the matrix-column $X^{T} Y$ :

$$
\left(X^{T} Y\right)^{-1} X^{T} Y=\left[\begin{array}{c}
0.045 \\
0.425 \\
1.083 \\
-0.343
\end{array}\right] .
$$

The obtained numerical results (8) allow to write the multiple regression equation:

$$
\tilde{Y}=0.045+0.425 \pi_{63}+1.083 \pi_{65}-0.343 \pi_{57} .
$$

In order to check the resulting model (9) to adequacy, it is necessary to calculate the $F$ - criterion (Fischer's criterion) value by $S_{Y}^{2}$ (total variance) and $S_{a d}{ }^{2}$ (remainder variance) (10):

$$
S_{Y}^{2}=\frac{1}{n-1} \sum_{i=1}^{n}\left(y_{i}-\bar{y}_{i}\right)^{2} ; S_{a d}^{2}=\frac{1}{n-1} \sum_{i=1}^{n}\left(y_{i}-\tilde{y}_{i}\right)^{2},
$$

where $n$-number of experiments;

$l=k+1-$ number of terms of the approximating polynomial;

$k$ - number of factors.

Thus, the calculated value of $F-$ criterion is equal to

$$
F_{\text {calculated }}=\frac{S_{a d}^{2}}{S_{Y}^{2}}=1.4 \text {. }
$$
is true

The multiple regression equation is significant with a significance level of $\alpha$, if the condition (12)

$$
F_{\text {calculated }}<F_{\alpha ; n-l, n-1},
$$

where $\quad F_{\alpha ; n-l, n-1}=4.34-$ critical value of the Fisher's distribution is found from table.

Therefore, we can conclude that the studied dependence is well described by the criteria $\pi_{63}, \pi_{65}$, $\pi_{57}$ with a given accuracy $[12 ; 13]$. Two mathematical models describing this process are obtained. The first model includes seven various factors (2). When substituting the numerical values of the criteria, we get the value of the resulting variable (13):

$$
\begin{aligned}
& \hat{Y}_{r a}=0.424+0.358 \cdot 3.300+0.879 \cdot 1.170+0.127 \cdot 0.700+1.600 \cdot 0.870-1.132 \cdot . \\
& \cdot 1.180-3.017 \cdot 0.060-1.026 \cdot 1.660=0.895
\end{aligned}
$$

The value of the resulting variable calculated using the obtained mathematical model is close to the experimental data and the value of the average resulting attribute is equal to 0.88. After determining the significant criteria, additional experimental studies were conducted, and the expression was obtained that includes three significant criteria $\pi_{63}, \pi_{65}, \pi_{57}(9)$. When substituting numerical values, we get (14):

$$
\tilde{Y}=0.045+0.425 \cdot 0.808+1.080 \cdot 1.025-0.343 \cdot 1.662=0.924 \text {. }
$$




\section{Results and discussion}

The special test stand was made for experiments (Fig.1). This test stand provides an opportunity to maintain the necessary conditions for the experiment and allows to install various acoustic-magnetic devices.

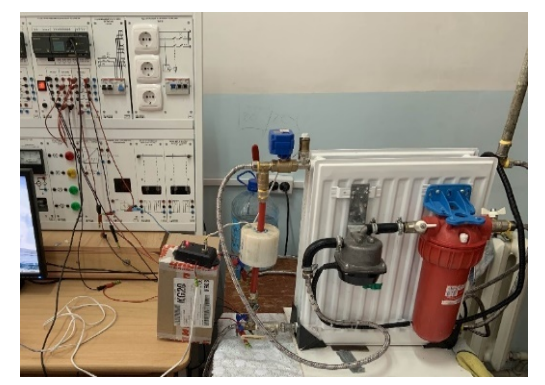

Fig. 1. Test stand for conducting experiments

The power supply of the electromagnetic system for reagent-free liquid treatment is provided from a three-phase alternating voltage source, which creates $\mathrm{AC}$ voltage in the resonant ultrasonic range of the resonant frequencies of the ferrite ring, which simultaneously allows the acoustic and rotating electromagnetic field to act on the liquids being processed, preventing salt deposits on pipelines.

There are various works describing the methods of acoustic or magnetic treatment of liquids [1417]. The authors Latva M., Inkinen J. reduce scale formation in the water supply system by $15 \%$ due to the use of magnetic treatment [14]. The authors Gabrielli C., Jaouhari R. [15] consider the main ways to increase the efficiency of magnetic processing, based on the optimization of process parameters: the power supply voltage, the flow velocity of the liquid, the pipe material, the treatment duration. As a result, it was possible to reduce the amount of scale by $18-20 \%$. Dular M., GriesslerBulc T. [16] indicate the possibilities of using cavitation for wastewater treatment and the control of various contaminants on surfaces. This method provides greater efficiency, but is very energyintensive. All these studies show a relatively low efficiency of the considered methods in comparison with the acoustic-magnetic geothermal water treatment, which allows achieving a reduction in scale formation by $80-85 \%$ due to the combined effect of acoustic and magnetic fields.

The obtained experimental data made it possible to build mathematical models, describing the process of scale formation under the influence of acoustic and magnetic fields on the geothermal water, which were used to verify the operability of the technique [3].

The calculated value of the resulting variable has a confidence interval of $0.904 \leq 0.924 \leq 0.944$ at the significance level of 0.05 . Testing the obtained models for adequacy proved the possibility of their practical application. The relative errors of approximation value are $4.98 \%, 4.28 \%$ for models (2) and (9). The obtained simplified model provides the possibility of its practical use for calculating the value of the resulting characteristic of the acoustic-magnetic treatment of geothermal water in the heat supply system of greenhouse complexes. Thus, the insignificant difference between the values obtained experimentally and using the generated mathematical models is demonstrated by the fact that the considered method [3], used to calculate the parameters of acoustic-magnetic devices, is working and can be used to calculate the parameters of the designed devices. Besides, using this technique, it will be possible in the future to calculate the parameters of the device that has optimal liquid treatment efficiency for pipes of various internal diameters. The experiments were carried out in order to verify the existing methodology for calculating the parameters of acoustic-magnetic devices considered in the works $[3 ; 5]$.

\section{Conclusions}

The paper considers experimental studies and processing of their results to verify the methodology for calculating the parameters of acoustic-magnetic devices.

During the study, all the necessary tasks for verification were completed: design and creation of special test stand for experiments; conducting experimental research; determination of the efficiency of the acoustic-magnetic device; the regression model making; determination of significant 
parameters; conducting an additional series of experiments for a new acoustic-magnetic device (its parameters are calculated using a verified method); comparison of the obtained experimental and calculated values.

According to the results of the study, it was found that the efficiency indicators of the devices installed in the greenhouse complex on pipes with various internal diameters differ insignificantly. This fact proves the efficiency of the used methodology for calculating the parameters of acoustic-magnetic devices and makes it possible to create devices with an acceptable value of the resulting indicator for pipes of the required range. It is planned to calculate the optimal parameters of the acoustic-magnetic devices, the efficiency of which does not depend on the diameter of the pipes used.

\section{References}

[1] Korzhakov A.V., Korzhakov V.E., Oskin S.V. A device for protection against the formation of deposits on the surfaces of pipelines of the heat supply systems. Patent of the Russian Federation No. 2635591, C23F 15/00 49/06 Application 2017100470. 09.01.2017. Publ. on 11/14/2017. Bul. No. 32 .

[2] Korzhakov A.V., Korzhakov V.E., Oskin S.V. A device for protection against the formation of deposits on the surfaces of pipelines of the heat supply systems. Patent of the Russian Federation No. 2641137, C02F 1/36; C02F 1/484 Application 2017100210. 09.01.2017. Publ. on 16/01/2018. Bul. No.2.

[3] Korzhakov A.V., Oskin S.V., Korzhakova S.A. Technique of creating new constructions of acoustic and magnetic devices based on theory of similarity as development of theory of experiment experiment. Materials of the XVIII International research and practical conference "Engineering for Rural Development", 2019. 1964 p.

[4] Gukhman A. A., Zaitsev A. A. Generalized analysis. Moscow: Factorial Publishing House, 1998. $304 \mathrm{p}$.

[5] Korzhakov A.V., Korzhakov V.E., Korzhakova S.A. Optimization of the Process of Acoustic and Magnetic Geothermal Water Treatment Through Simulation in CoDeSys. In Lecture Notes in Electrical Engineering (Vol. 641 LNEE, pp. 1019-1033). Springer.

[6] Johnson N., Leone F. Statistics and experimental design. Moscow: World, 1980. 511 p.

[7] Ermakov S. M., Brodsky V. Z., Zhiglyavsky A. A. etc. Mathematical theory of experiment planning. Moscow: Fizmatlit, 1983. 392 p.

[8] Montgomery D. K. Experiment Planning and data analysis. London: Shipbuilding, 1980. 380 p.

[9] Nalimov V. V. Theory of experiment. Moscow: Nauka, 1971. 208 p.

[10] Adler Y. P., Granovsky Y. V. Planning an experiment in the search for optimal conditions. Moscow: Nauka, 1971.

[11] Antonov S. N., Adashev A. I., Sharipov I. K. etc. Devices of magnetic water treatment. Stavropol: Agrus of Stavropol State agrarian university, 2014. 220 p.

[12] Ayvazyan S. A., Mkhitaryan V. S. Applied statistics and fundamentals of econometrics. Moscow: UNITY, 1998. 1023p.

[13] Kulikov E.I. Application statistical analysis. Moscow: Telecom, 2008. 464 p.

[14]Latva M., Inkinen J., Rämö J. et al. Studies on the magnetic water treatment in new pilot scale drinking water system and in old existing real-life water system. Journal of Water Process Engineering 9, 2016, pp. 215-224.

[15] Gabrielli C., Jaouhari R., Maurin G, Kedda M. Magnetic water treatment for scale prevention. Water Research 35, 2001, pp. 3249-3259.

[16]Dular M., Griessler-Bulc T., Gutierrez-Aguirre I. et al. Use of hydrodynamic cavitation in (waste)water treatment. Ultrasonics Sonochemistry 29, 2016, pp. 577-588.

[17] Gevari M., Abbasiasl T., Niazi S. Direct and indirect thermal applications of hydrodynamic and acoustic cavitation: A review. Applied Thermal Engineering 171, 2020. 